\title{
Regression of pulmonary metastases with long term remission in a patient with renal carcinoma
}

\author{
MENASHE M RAHIMA, JOSHUA BARZILAY \\ From the Department of Oncology, Beilinson Medical Center, Petah Tikva, Israel
}

Despite the poor prognosis of patients with renal carcinoma and pulmonary metastases instances of prolonged survival with regression of the lung lesions have been reported. We present one such case, in which multiple cannon ball lesions seen on the chest radiograph resolved completely for nine years and a prolonged clinical remission was achieved after hormone treatment and chemotherapy.

\section{Case report}

A 55 year old woman was admitted to hospital for evaluation of a left renal mass. A chest radiograph on admission showed a calcified paratracheal lymph node and signs of antecedent tuberculosis. The blood chemistry was normal and liver and bone scans yielded normal results.

In January 1973 she underwent a left nephrectomy. A large tumour was found in the lower pole of the kidney and histological examination showed a characteristic picture of clear renal carcinoma with invasion of the blood vessels. She was referred to our institute for treatment and in March 1973 she received a course of radiation to the renal bed with a total dose of 4000 rads (cGy).

Two months after treatment the patient began complaining of a non-productive cough and a further chest radiograph (fig 1) showed multiple "cannon ball" lesions $2-4 \mathrm{~cm}$ in diameter characteristic of metastatic renal cell carcinoma. Hormone treatment with medroxyprogesterone acetate $200 \mathrm{mg}$ intramuscularly five times a week and chemotherapy with vinblastine $0.1 \mathrm{mg} / \mathrm{kg}$ intravenously once a week was administered. Over the following four months the patient reported subjective improvement and regression of the tumor nodules on the chest radiograph was noted. In the sixth month of treatment there was a complete disappearance of all pulmonary lesions (fig 2) and the patient felt well.

Medroxyprogesterone $200 \mathrm{mg}$ intramuscularly twice a week and vinblastine $0.1 \mathrm{mg} / \mathrm{kg}$ intravenously once a week were continued until May 1977 after which she received only medroxyprogesterone $200 \mathrm{mg}$ twice weekly. She remained symptom free, and repeat chest radiography at three month intervals showed no evidence of tumour. At the end of the eighth year after nephrectomy treatment was discontinued. One year later left hilar adenopathy and opacification of the left lower lobe was noted on a routine chest radiograph, but the patient refused treatment. Over

Address for reprint requests: Dr $M$ Rahima, Department of Oncology, Beilinson Medical Center, Petah Tikva, Israel 49100.

Accepted 1 September 1984

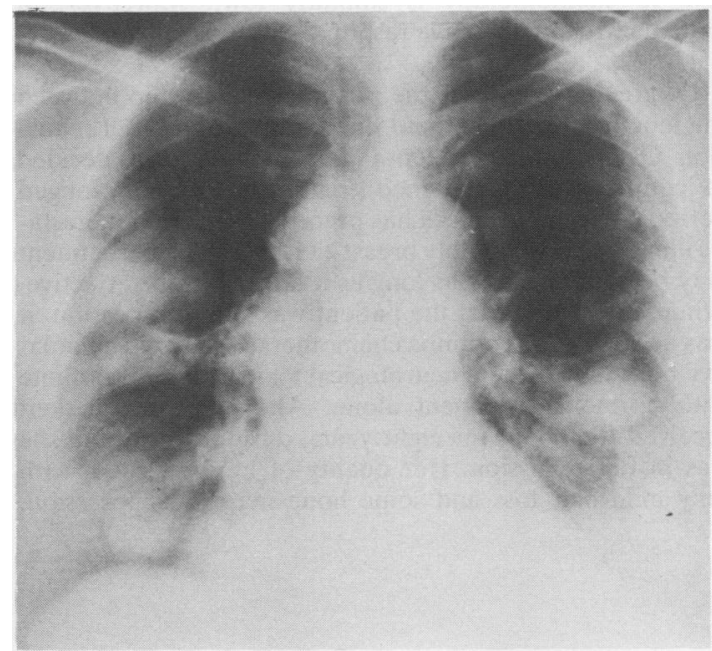

Fig 1 Chest radiograph showing multiple metastatic lesions.

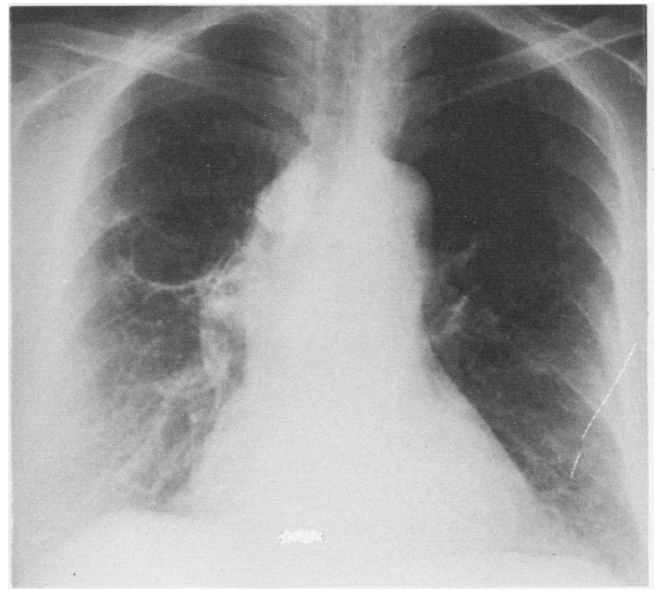

Fig 2 Chest radiograph six months after treatment was begun showing resolution of the lesions. There is calcification in a right paratracheal gland.

the following year the pulmonary lesions multiplied and cerebral metastases were diagnosed before her death in December 1982, 10 years after nephrectomy. 


\section{Discussion}

In the case presented here complete clinical and radiological regression of metastatic renal carcinoma in the lungs lasting nine years was observed. The temporal relationship between the onset of treatment and the tumour regression suggests that in this case the disease was brought into remission by the treatment. Complete disease control has rarely been reported in renal carcinoma. The response rate to hormone treatment is at best only $15 \%,{ }^{1}$ with most series reporting no objective responses at all ${ }^{2}$; and the response rate to chemotherapy is similarly low. ${ }^{3}$ Moreover, in patients with an objective response this is usually only partial.

Of greater interest is the possible relationship between the length of treatment and the duration of the full remission. Owing to the excellent initial response it was decided to continue with combined treatment for a prolonged period. Such an approach has precedents in other metastatic illnesses, most notably breast cancer, in which treatment may be continued for as long as it appears to be effective. When after four years the patient was still in remission, it was decided to discontinue chemotherapy to avoid cumulative bone marrow and neurological toxicity and to continue with hormone treatment alone. Altogether the patient received treatment for eight years, during which time she was in full remission. Her quality of life was good, with only mild hair loss and some bone marrow suppression.
The disease recurred within one year of the termination of treatment, suggesting that it had been controlled by the treatment and, more specifically, by the hormone therapy. It should, however, be noted that assessment of any treatment in renal carcinoma is complicated by the peculiar tendency of this disease to remain dormant for many years or to regress spontaneously without treatment. In one study of patients with prolonged survival after nephrectomy, $11 \%$ developed metastatic lesions after 10 years. ${ }^{4}$ Cases documenting spontaneous tumour regression have likewise been reported..$^{5}$ The most common site in both these instances has been the lungs, so that spontaneous regression with late recurrence cannot be completely excluded in the present case.

\section{References}

1 Bloom HJ. Hormone induced and spontaneous regression of metastatic renal cancer. Cancer 1973;32:1066-77.

2 deKernion JB. Treatment of advanced renal cell carcinoma traditional methods and innovative approaches. J Urol 1983;130:2-7.

3 Raghavaiah NV. Hormone treatment of advanced renal cell carcinoma. Urology 1982;19:123-6.

4 McNichols DW, Segura JW, DeWeerd JH. Renal cell carcinoma: long term survival and late recurrence. J Urol 1981;126: 17-23.

5 Snow RM, Schellhammer PF. Spontaneous regression of metastatic renal cell carcinoma. Urology 1982;20: 177-81. 